\title{
Impressões qualitativas em espaços urbanos noturnos por meio de ambientes virtuais imersivos
}

\author{
Qualitative impressions in urban spaces at night through immersive virtual \\ environments
}

Ítalo Pereira Fernandes ${ }^{[a]}$, Norberto Corrêa da Silva Moura ${ }^{[0]}$, António Aguiar Costa ${ }^{[b]}$

\author{
[a] Universidade de São Paulo (USP), São Paulo, SP, Brasil \\ [b] Instituto Superior Técnico Lisboa, Lisboa, Estremadura, Portugal
}

\section{Resumo}

Pesquisas no âmbito da iluminação focam no aspecto quantitativo e físico dos efeitos lumínicos e seu desempenho na relação usuário-ambiente. Assim, estudos sobre os aspectos subjetivos da iluminação e sua influência na percepção e no usufruto do espaço são crescentes, porém existem poucos experimentos para avaliar impressões subjetivas dos ambientes noturnos. 0 presente artigo tem por objetivo mapear qualidades ambientais do espaço urbano localizado na cidade de João Pessoa-PB por meio do conceito de atmosfera percebida, cuja categorização de palavras descreve qualitativamente o ambiente, utilizando o ambiente virtual tridimensional como suporte para criação e avaliação de cenários noturnos. Nesse sentido, foram simulados, em realidade virtual, dois projetos de iluminação: uma paisagem noturna existente e uma proposta de intervenção como alternativa, a fim de gerar avaliações pelos usuários. Os resultados mostram que existem qualidades subjetivas diferentes nos espaços, relacionadas com questões de segurança e sociabilidade do ambiente, o que reflete a importância dessas avaliações subjetivas, e demonstram o contributo da realidade virtual como aspecto inovador nesse processo. Contribuições do artigo dizem respeito à participação coletiva no processo de projeto e aplicação da metodologia em contextos em que a iluminação é fundamental, como espaços públicos de conjuntos habitacionais.

Palavras-chave: Iluminação. Percepção ambiental. Realidade virtual.

\section{Abstract}

Research in the lighting field usually has focused on the quantitative and physical aspects of light and its performance for the user-environment relationship. Despite the growing research on subjective aspects of lighting and its influence on the perception of the built environment, experiments evaluating the impressions of nocturnal environments are still few. This article consisted in the mapping of projected environmental qualities of urban spaces in the city of João Pessoa-PB based on the concept of perceived atmosphere, which proposes words that qualitatively describe an environment. The experiment employed virtual reality to support the creation of virtual night scenes, simulating lighting projects, such as the existing night situation as the first

IPF é arquiteto e urbanista, mestre em Tecnologia da Arquitetura, e-mail: italo@live.com NCSM é arquiteto e urbanista, doutor em Tecnologia da Arquitetura, e-mail: betomoura@usp.br AAC é engenheiro civil, PhD em Engenharia Civil, e-mail: aguiar.costa@tecnico.ulisboa.pt 
scene and a new lighting intervention as a second alternative, in order to generate comparative evaluations. The results showed that there are different qualities in both spaces, mainly related to safety and sociability issues, reflecting the importance of adequate assessments of urban perceptions as well as virtual reality as an innovative tool. The contributions of this article include the possibility of replication of the methodology in new contexts where public lighting plays a key role, such as public spaces in housing developments and collective participation in the design process.

Keywords: Lighting design. Environmental perception. Virtual reality.

\section{Introdução}

A iluminação é essencial para qualidade de vida nos centros urbanos, visto que permite a ocupação, o deslocamento e o usufruto dos espaços públicos pela população no período noturno. Aliado às questões técnicas e subjetivas, o cenário iluminado valoriza patrimônios históricos, embeleza a paisagem natural, conta história por meio dos seus percursos e traz uma melhor imagem da cidade, com benefícios ao usuário do espaço, com a possibilidade de tornar-se um fator de atratividade do turismo, comércio e lazer.

Desde a década de 1960, a cultura do automóvel vem ditando as normas e recomendações a respeito de como deve ser iluminado o espaço urbano, abordando questões econômicas das fontes de luz (Mascaró, 2006). Assim, a velocidade e a escala dos carros, em detrimento dos pedestres nesses locais, são a abordagem mais recorrente (street lighting), desconsiderando diferenças e particularidades em prol da eficiência e baixo custo. 0 resultado desse cenário foi uma iluminação uniforme e pouco refinada, cuja luz incide sempre nas vias carroçáveis, causando impactos, por exemplo, sombras projetadas sobre as calçadas, o que gera impressão de insegurança aos pedestres, e também problemas ambientais, como a poluição luminosa e o ofuscamento.

O desenvolvimento de políticas de iluminação sob o ponto de vista qualitativo, priorizando as necessidades funcionais e emocionais dos usuários que circulam pelos espaços urbanos, começa a ser estimulado a partir do potencial turístico e econômico das cidades de Lyon e Paris, como consequência de um plano diretor de iluminação em fins da década de 1980 e a relação entre os habitantes e os locais da cidade (Hong, 2007).

Assim, o planejamento da luz em espaços urbanos passa a ser entendido não só como iluminação de pontos isolados e vias de tráfego, mas um espaço organizado arquitetonicamente, que privilegia ambiências e atmosferas - um meio físico, estético e psicológico - , ou seja, refletindo o modo de viver, as necessidades e os valores produzidos pela cidade e identificados pela comunidade (Gonçalves, 2005).

Ao passo que os aspectos subjetivos e a influência da iluminação na relação entre a cidade e o usuário são relevantes nesse processo, acredita-se que os ambientes para os quais um projeto luminotécnico deverá ser concebido podem se beneficiar das impressões das pessoas ainda nos primeiros estágios de design, por meio da participação coletiva e das avaliações centradas nas necessidades dos usuários no espaço (User Centered Design) (Heydarian et al., 2015a, 2015b), a fim de melhorar o produto final, promovendo a identificação dos valores e modos de vida da população.

No entanto, alternativas de cenários a serem avaliados esbarram em obstáculos logísticos e financeiros, como custos elevados no processo de testes em ambiente físico, o que justifica a utilização de ambientes computacionais, como a realidade virtual. Esta tem como propósito permitir ao usuário a interação com ambientes virtuais tridimensionais em tempo real, desenvolvendo-se um senso de presença e de imersão com o espaço ao explorá-lo (Tori \& Kirner, 2006).

A aplicação dessas ferramentas computacionais na simulação de ambientes virtuais possui desdobramentos em diferentes áreas, por exemplo, habitações de interesse social. Diferentes soluções de projeto podem ser avaliadas ainda nas fases iniciais do processo, a partir de questões como percepção de segurança e o conforto nos espaços públicos dos conjuntos habitacionais, tendo em vista a capacidade de interação e imersão nesses ambientes por parte dos usuários. Nesse sentido, os ambientes virtuais podem inserir a comunidade na tomada de decisões e contribuir para a compreensão das expectativas dos moradores e as intenções do projeto. 
0 presente artigo pretendeu analisar impressões dos usuários em ambientes externos noturnos por meio da teoria da atmosfera percebida, desenvolvida por Vogels (2008), e utilizando a realidade virtual como mediador no processo de simulação e exploração de cenários de iluminação. Dessa forma, foi possível gerar reflexões críticas a respeito do processo a fim de estabelecer diretrizes em longo prazo para a pesquisa, a qual foca na possibilidade de utilização dos dados da participação coletiva como forma de auxiliar no processo de planejamento do projeto, batizado de iluminação urbana 2.0 por Bessete (2011 apud Casciani, 2013), em referência a WEB 2.0.

\section{Avaliação e percepção da luz}

Tradicionalmente, pesquisas no âmbito da iluminação têm predominância no aspecto quantitativo e físico dos efeitos da luz e sua performance para a relação usuário-ambiente. Aliado a isso, pouca pesquisa existe sobre os aspectos subjetivos da iluminação e sua influência na percepção e no usufruto do ambiente construído. Essa dualidade de estudos tem a ver com o histórico das atribuições profissionais, no qual os engenheiros eram responsáveis por conduzir os estudos técnicos, enquanto a inserção de arquitetos e designers no processo de projeto começaram a questionar os aspectos de percepção ambiental e a pesquisar a influência da iluminação na percepção do usuário.

\section{Aspectos subjetivos em iluminação}

De um lado, temos o avanço das tecnologias de iluminação e os estudos relacionados a qualidades técnicas e quantitativas, como medições fotométricas, temperatura da cor, custo, benefício e eficiência para a compreensão da influência da iluminação na performance do usuário (Knight, 2010; Fotios et al., 2005). Incluem-se ainda estudos nos quais usuários expostos a fontes luminosas são avaliados a fim de descobrir a quantidade de luz necessária para fornecer segurança física (detecção de obstáculos e reconhecimento facial) e conforto visual, além de outros aspectos, como depreciação de fontes luminosas, entre outros (Boyce et al., 2000).
Tem-se, por outro lado, características qualitativas dos espaços iluminados que provocam sensações e estabelecem conexões com quem se apropria em um fluxo de informações geradas pelo ambiente ao usuário. Aqui estão inseridas pesquisas que se propõem a entender como as pessoas percebem e avaliam o espaço, bem como sua relação com o ambiente iluminado, a partir de uma abordagem em termos psicológicos, sociais, subjetivos (Veitch, 2001; Custers et al., 2010).

Estudos relacionados com a psicologia ambiental do espaço sugerem que a iluminação exerce influência nas emoções, estados e comportamentos dos usuários, como tensão, relaxamento, prazer, desconforto, mistério e coerência (Vogels, 2008; Kaplan, 1992), além do fato de gerar diferentes impressões visuais e espaciais do espaço, dependendo da distribuição da luz, como ordem, altura e profundidade (Flynn et al., 1973; Lindh, 2012).

Flynn et al. (1979), por exemplo, desenvolveram um experimento que procura relacionar atributos de iluminação — luz não uniforme, brilho, luz periférica - e as consequências das impressões visuais e espaciais nos diferentes usuários, identificando alguns aspectos, como impressão de espaço maior ou confinado e clareza visual. Com base no seu estudo, foram propostas ainda estratégias de iluminação e seus possíveis efeitos no usuário, como a utilização de luz não uniforme e dimerizada para gerar sensação de um espaço privativo, íntimo.

Para o presente artigo, foi utilizado como base teórica o conceito de atmosfera percebida, desenvolvida por Vogels (2008), por entender a noção da percepção como uma avaliação do ambiente relacionado ao efeito esperado que dado espaço exerce, não necessariamente correspondendo ao real estado emocional. Essa autora justifica que, apesar das emoções flutuarem de forma repentina, as qualidades projetadas sobre o ambiente permanecem mais estáveis.

\section{Processos e produtos no projeto de iluminação}

O processo de projeto ocorre por meio de associações mentais das intenções do lighting designer e as possibilidades que a iluminação pode causar em dado ambiente. Os chamados "efeitos de luz" (Skarlatou, 2010) - definição de efeitos que são resultado da interação entre a luz e espaço, por exemplo, uplight, downlight, luz 
difusa, luz indireta - são imaginados pelo projetista, que combina características técnicas, como temperatura de cor e brilho, em uma composição espacial.

A partir da dualidade entre a técnica e a subjetividade, é comum os profissionais recorrerem a programas específicos de cálculo luminotécnico para verificação do projeto em termos de brilho, contraste, uniformidade, a fim de alcançar níveis adequados ao local (Cypriano, 2013). A composição dos efeitos de luz deverá trazer características estéticas, além de encargos psicológicos que causam nos usuários sensações e percepções em dado ambiente. Por não ser a luz uma matéria palpável, sua percepção é complexa, o que dificulta encontrar um meio que represente e comunique as intenções do projeto.

0 resultado da iluminação, que perpassa desde a criação de conceitos, referências culturais, ideais e crenças do projetista, necessidades físicas e psicológicas dos usuários até o conhecimento técnico do campo de atuação, é observado em produtos do seu pensamento, como desenhos, conceitos e palavras, que serão organizados a fim de transmitir da melhor forma ao cliente o que se pretende fazer.

Atualmente, a avaliação dos projetos tem um cariz essencialmente visual, na qual a representação dos efeitos de luz e de sombra é demonstrada por meio de recortes dos efeitos de luz resultantes das luminárias em catálogo de fabricantes, imagens computadorizadas, privilegiando-se pontos de vista específicos, ou desenhos a mão, além de textos explicando o conceito desenvolvido.

Do ponto de vista qualitativo, existem diferenças entre perceber um cenário estático por intermédio de fotografias (Michel, 1996 apud Lindh, 2012, p. 50) e a possibilidade de caminhar e ter a experiência de explorar o espaço, pois essa relação contextual e as diferentes visões do ser humano, ângulos e distâncias são importantes para avaliação dos ambientes.
Outra maneira de explicar os efeitos que se pretende atingir com o projeto de iluminação é a utilização de mockups, que é uma ferramenta essencial para a compreensão do projeto. Nesse caso, é construído um modelo em escala natural do ambiente que se está estudando, cujo projeto é reproduzido de acordo com as especificações para avaliação dos efeitos de luz e sua relação com o espaço, as superfícies e os seus materiais (Figura 1).

A aplicação do mockup é recorrente em áreas que precisam realizar protótipos antes da finalização do produto, como forma de gerar melhores resultados na otimização dos gastos, que vão desde a indústria automobilística até a arquitetura e urbanismo.

Um exemplo é a construção de mockups em ambientes hospitalares. Segundo Dunston et al. (2011), com vistas a reduzir custos e melhorar o produto final, a inspeção realizada nesses ambientes pelos atores envolvidos no processo - equipe médica, arquitetos e administradores - possibilita identificar aspectos funcionais e qualitativos a serem revistos. Tendo em vista a complexidade de locais, como salas de cirurgia, ou a repetição de módulos, como quartos de pacientes, erros podem ser identificados antes da real construção. Essa justificativa se aplica ao planejamento de usinas nucleares e à construção de trechos de fachadas para avaliação da performance e métodos construtivos (Whisker et al., 2003; Maing, 2012).

0 uso de ambientes virtuais tridimensionais se apresenta como alternativa à criação de mockups, cuja realidade virtual é um meio de acesso, na medida em que o processo de exploração do espaço pretende ser similar, menos custoso, gerando resultados semelhantes. Esses cenários virtuais são representações sintéticas da realidade ou criações da imaginação, viabilizados pela tecnologia computacional, cujas pesquisas datam desde 1960 e vêm permitindo a troca de informações

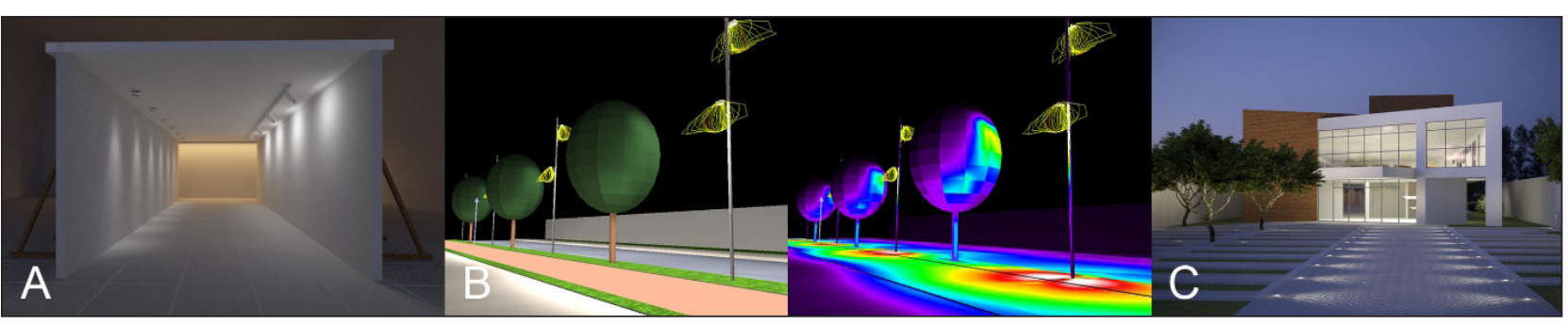

Figura 1 - Diferentes produtos do projeto de iluminacção para avaliação da qualidade lumínica. (A) de ambiente interno; (B) Software de cálculo luminotécnico; (C) Maquete eletrônica

Fonte: Elaboração própria.

urbe. Revista Brasileira de Gestão Urbana (Brazilian Journal of Urban Management), 2018 jan./abr., 10(1), 95-110 
entre o sujeito e o meio virtual em tempo real, principalmente por meio de estímulos visuais (Tori \& Kirner, 2006).

Os ambientes virtuais fazem parte do escopo da arquitetura desde os anos de 1990, por meio de estudos técnicos, como processamento de dados e visualizações realísticas de superfícies em edifícios (Bullinger et al., 2010). Com o avanço da capacidade gráfica dos computadores pessoais e da indústria do videogame, novas funcionalidades e novos cenários mais sofisticados puderam ser criados, possibilitando pesquisas em campos específicos que exercem influência sobre a arquitetura, como melhorias em sombra, luz e vegetação (Mantler et al., 2003 apud Bullinger et al., 2010), além da interação e visualização de projetos em BIM (Building Information Modelling) pelos projetistas (Yan et al., 2011).

Assim, permite a criação de modelos digitais para aplicações diversas, como avaliação da linha de montagem de um automóvel (Sá \& Zachmann, 1999), projeto e ensino de arquitetura (Dunston et al., 2011; Angulo \& Velasco, 2014), e também como forma de auxiliar o processo de projeto em paisagismo e a participação de agentes importantes - arquiteto, público, administradores - no redesenho de uma praça (Ball et al. 2007; Drettakis et al., 2007).

Em iluminação, a realidade virtual é utilizada para analisar a preferência de usuários quanto ao controle lumínico em ambiente de escritório (Heydarian et al., 2015a, 2015b). Portanto, o papel desses recursos é garantir que as ideias traduzidas em imagens e textos no processo de planejamento sejam transformadas em realidade antes da finalização do projeto.

A avaliação com base em mockups enfrenta obstáculos para sua realização, envolvendo custo de material e instalação, procedimentos burocráticos e logísticos. Caso o ambiente estudado seja um espaço interno, por exemplo, quartos de hospitais, a variação do custo de construção pode ser de centenas de milhares de dólares, desde a locação de galpão, equipamentos, construção do espaço e demolição após uso (Dunston et al., 2011).

Em iluminação pública, a reprodução do espaço para avaliação em escala natural necessita de intervenções no próprio local. Em espaços urbanos tombados, é imprescindível a autorização de órgãos responsáveis pela gestão do espaço público - instituto do patrimônio histórico (IPHAN e superintendências regionais), prefeitura, distribuidor de energia e entes particulares - , além da instalação dos equipamentos especificados no projeto por determinado período de tempo.

A realidade virtual é uma opção aos mockups (Tabela 1), principalmente quando relacionada à redução de custos, à maior capacidade de modificações no processo de projeto e à predominância da percepção visual sobre outros sentidos, como interações táteis com superfícies (Maing, 2012).

Porém, alguns fatores podem dificultar a aceitação da ferramenta. A imersão do usuário, navegação pelo espaço e manipulação de objetos virtuais são feitos por meio de dispositivos auxiliares, como óculos estereoscópicos (head-mounted display - HMD) e controles. Longa exposição e erros, por exemplo, tremulações de imagem, rastreamento da posição do usuário e a sua atualização no cenário virtual, podem causar desconforto e mal-estar, chamado "simulator sickness" (Carvalho et al., 2011; Llorach et al., 2014). Outro aspecto diz respeito ao manuseio do aparato tecnológico por parte dos usuários, cuja faixa etária e familiaridade com videogames podem influenciar no uso da realidade virtual (Portman et al., 2015).

Mesmo ao levar em consideração as limitações e fragilidades da ferramenta digital, seja no desempenho dos dispositivos de imersão, seja na capacidade de processamento de dados, seja no manuseio do aparato tecnológico - o que pode gerar desconforto e influenciar nos resultados da pesquisa - , o ambiente virtual tridimensional pode fornecer o suporte adequado para a reprodução de um cenário real ao qual será adicionada a iluminação e no qual é permitido ao usuário explorar e interagir com o espaço por meio de uma interface virtual e equipamentos auxiliares. Assim, terá a possibilidade de gerar avaliações sobre suas impressões do cenário desenvolvido com base no projeto luminotécnico, além de contribuir como mediador no planejamento de uma melhor experiência noturna no espaço urbano.

\section{Materiais e métodos}

O objetivo do presente estudo é mapear qualidades ambientais a partir de impressões subjetivas dos usuários inseridos no espaço urbano noturno. 0 ambiente simulado digitalmente possibilita a exploração do espaço e a avaliação de cenários por meio do questionário da atmosfera percebida, o 
Tabela 1 - Quadro comparativo entre dois modelos de simulação: mockup e realidade virtual

\begin{tabular}{|c|c|c|c|}
\hline & DESCRIC̣̃̃O & MOCKUP & REALIDADE VIRTUAL \\
\hline \multirow[t]{4}{*}{ CICLO DE VIDA } & espaço físico & $\bullet$ & $\circ$ \\
\hline & despesas iniciais de construção & $\bullet$ & $\bullet$ \\
\hline & retrabalho & $\bullet$ & $\bullet$ \\
\hline & demolição & $\bullet$ & $\circ$ \\
\hline \multirow[t]{3}{*}{ CARACTERÍSTICAS } & acessórios & $\circ$ & - (hmd, mouse, controle) \\
\hline & interação sujeito-objeto & $\bullet$ & $\bullet$ \\
\hline & colaboração entre disciplinas (AEC) & - (próximo à construção final) & - (implementação nas primeiras fases) \\
\hline \multirow[t]{5}{*}{ PERCEPCฺ̃̃ES E NECESSIDADES } & tátil & $\bullet$ & 0 \\
\hline & visual & $\bullet$ & $\bullet$ \\
\hline & sonora & $\bullet$ & • (sonorização 3D) \\
\hline & usabilidade & $\circ$ & - (equipamentos auxiliares) \\
\hline & compreensão do espaço & $\bullet$ & - (equipamentos auxiliares) \\
\hline \multirow[t]{4}{*}{ OUTROS PROCEDIMENTOS E CUSTOS } & autorização de órgãos responsáveis & - (intervenções em ambientes reais) & $\circ$ \\
\hline & locação de equipamentos & $\bullet$ & $\circ$ \\
\hline & aquisição de softwares e hardwares & $\circ$ & $\bullet$ \\
\hline & & LEGENDA & - (SIM) ○ (NÃO) \\
\hline
\end{tabular}

Fonte: Elaboração própria.

qual atribui descritores às qualidades projetadas e esperadas sobre o ambiente (Figura 2).

0 experimento considera a criação de alternativas de cenário por intermédio do uso da realidade virtual, tendo em vista a viabilidade de execução da pesquisa. Na verdade, a utilização da realidade virtual permite a apropriação do espaço tridimensional ao oferecer maior controle sobre variáveis difíceis de serem controladas em condições reais, como a criação de cenário de luz em espaço público e a possibilidade de receber avaliações dos usuários e suas impressões — configurando uma avaliação pré-ocupação - em curto período de tempo.

De acordo com Vogels (2008), foram selecionados os termos que descrevem aspectos qualitativos dos ambientes, como espaços ora relaxantes, ora ameaçadores, ora inspiradores, ora deprimentes, os quais foram captados pelos questionários. Foram selecionados 22 dos 38 termos da atmosfera percebida, analisados eagrupados em quatro dimensões: aconchego (coziness), ânimo (liveliness), tensão (tenseness) e distanciamento (detachment).

Para a execução deste experimento, procedeu-se à análise e à adequação dos termos da língua original em holandês para o português, tendo em vista a tradução pela autora apenas para o inglês (Tabela 2).

A primeira dimensão chamada de aconchego (coziness) abrange termos que descrevem o ambiente como tranquilo, agradável, relaxante, romântico, seguro e acolhedor e estão ligados diretamente a sensações positivas do ambiente. Quanto à dimensão ânimo (liveliness), ela compreende termos qualitativos, por exemplo, vivaz, estimulante, agitado, alegre, inspirador e sociável, sendo descritores referidos à vitalidade do local. As características da dimensão de tensão (tenseness) dos ambientes, onde foram considerados termos como estressante, deprimido, assustador, intimidador, desconfortável e tedioso, são ligadas a aspectos negativos do espaço.

Por fim, última dimensão, distanciamento (detachment), inclui termos como acessível, familiar, exclusivo e desinibido, tendo aspectos relacionados ao comportamento e à impressão do espaço que subjugam o usuário (Tabela 3). Um ambiente é considerado exclusivo ao entender que ele pressupõe padrões de comportamento e status. Um exemplo é o ambiente em uma loja de marca que reflete certa classe social e o poder aquisitivo dos consumidores. 0 mesmo pode 


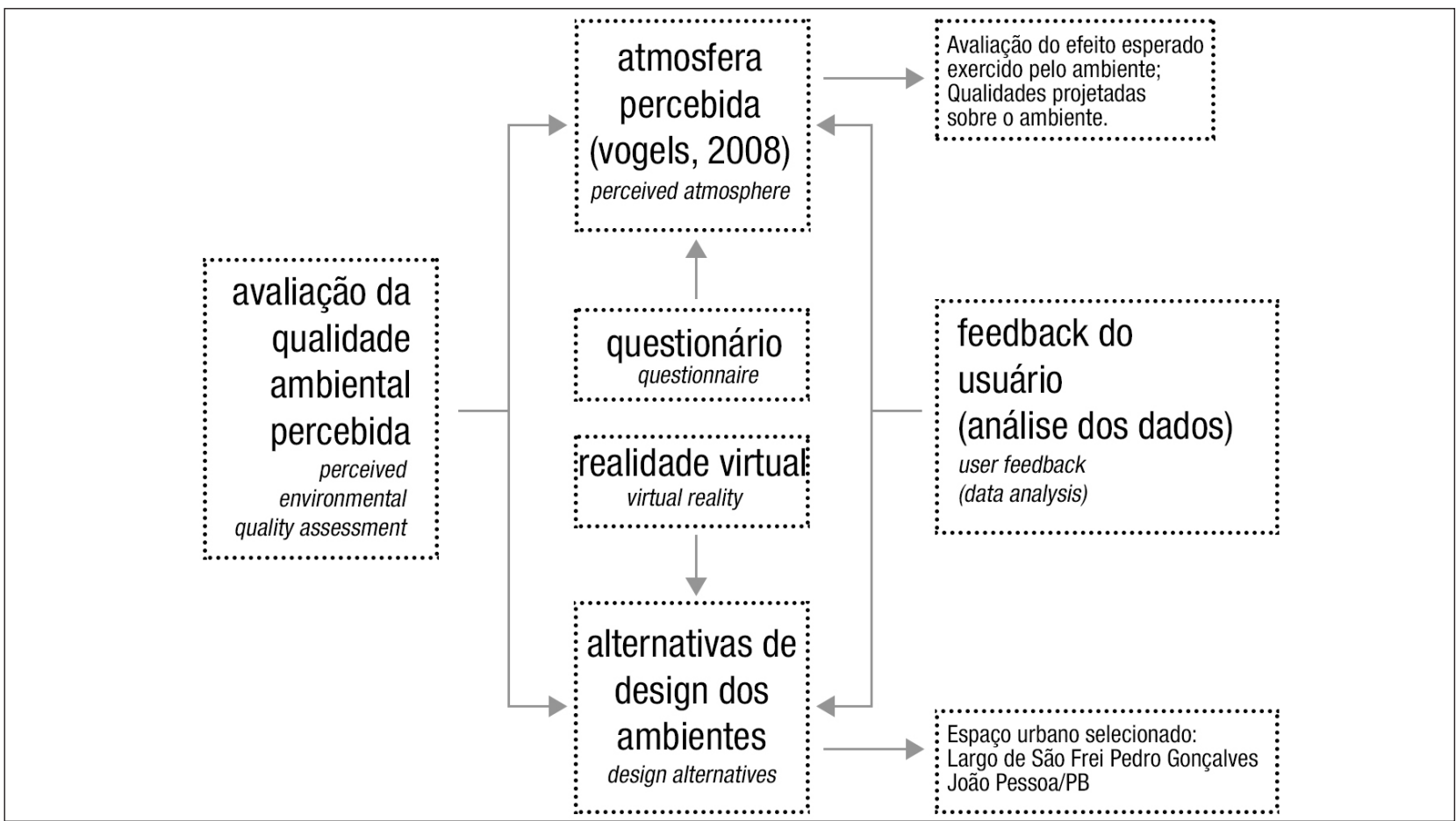

Figura 2 - Mapa conceitual do experimento descrevendo as rotinas metodológicas desenvolvidas no trabalho Fonte: Elaboração própria.

Tabela 2 - Termos da atmosfera percebida de Vogels. (Em negrito os selecionados para o experimento)

\begin{tabular}{|c|c|c|c|c|c|}
\hline Holandês & Inglês & Português & Holandês & Inglês & Português \\
\hline afstandelijk & detached & inamistoso & levendig & lively & vivaz \\
\hline beangstigend & terrifying & assustador & luxueus & luxurious & luxuoso \\
\hline bedompt & musty & abafado & mysterieus & mysterious & misterioso \\
\hline bedreigend & threatening & intimidador & ongedwongen & uninhibited & desinibido \\
\hline behaaglijk & cozy & acolhedor & ongemakkelijk & uncomfortable & desconfortável \\
\hline beklemmend & oppressive & opressivo & onrustig & restless & agitado \\
\hline deprimerend & depressed & deprimido & ontspannen & relaxed & relaxado \\
\hline enerverend & exciting & excitante & persoonlijk & personal & íntimo \\
\hline formeel & formal & exclusivo & omantisch & romantic & romântico \\
\hline gastvrij & hospitable & acolhedor & ruimtelijk & spatial & amplo \\
\hline geborgen & safe & seguro & rustgevend & tranquil & tranquilo \\
\hline gemoedelijk & pleasant & agradável & saai & boring & tedioso \\
\hline gespannen & tense & estressante & sloom & lethargic & apático \\
\hline gezellig & pleasant & sociável & stimulerend & stimulating & estimulante \\
\hline inspirerend & inspiring & inspirador & toegankelijk & accessible & acessível \\
\hline intiem & intimate & familiar & vijandig & hostile & hostil \\
\hline Kil & chilly & ermo & vrolijk & cheerful & alegre \\
\hline Knus & cozy & aconchegante & warm & warm & caloroso \\
\hline Koud & cool & frio & zakelijk & business & pragmático \\
\hline
\end{tabular}

Fonte: Adaptado de Vogels (2008). 
ocorrer em espaços urbanos, em intervenções, como cerramento de áreas públicas por meio de grades.

\section{Área de estudo}

O espaço do Largo de São Frei Pedro Gonçalves (LSFPG), localizado em João Pessoa-PB, foi o selecionado como objeto de estudo. Situa-se no bairro do Varadouro, local de fundação da cidade a partir do Rio Sanhauá, e faz parte da chamada "cidade baixa" por causa da sua topografia acidentada. Tombado em 2009 pelo Instituto do Patrimônio Histórico e Artístico Nacional (IPHAN), possui cerca de $7 \mathrm{mil} \mathrm{m}^{2}$ de área (largo e edificações adjacentes). Possui como edificações importantes a Igreja, que dá nome ao Largo, um antigo hotel da cidade, além de um conjunto de casarios onde funcionam desde instituições a restaurantes.

Os participantes eram solicitados a caminhar pelo ambiente virtual em cada um dos cenários para, em seguida, preencher um questionário a respeito das qualidades percebidas do espaço e sua atmosfera percebida apenas nos cenários noturnos.

\section{Modelo e recursos de imersão virtual}

0 modelo tridimensional da área urbana (Figura 3) foi reproduzido de acordo com as dimensões e texturas do ambiente físico, a fim de aproximar o ambiente virtual da realidade. Também foi criado com o objetivo de implantação de cenas noturnas, nas quais a iluminação desempenha papel importante na relação do usuário com as qualidades percebidas do espaço e suas impressões subjetivas.

Para o estudo em causa, foram configurados três cenários com distintas soluções de iluminação: um cenário diurno, chamado de Cenário 0 , e dois cenários noturnos, o Cenário 1 reproduzindo a situação existente

Tabela 3 - Resumo das quatro dimensões e respectiva abrangência de termos

\begin{tabular}{cccc}
\hline aconchego & ânimo & tensão & distanciamento \\
\hline acolhedor & vivaz & estressante & acessivel \\
seguro & estimulante & assustador & familiar \\
romântico & agitado & deprimido & exclusivo \\
relaxado & alegre & intimidador & desinibido \\
agradável & inspirado & desconfortável & \\
tranquilo & sociável & tedioso & \\
\hline
\end{tabular}

Fonte: Elaboração própria.

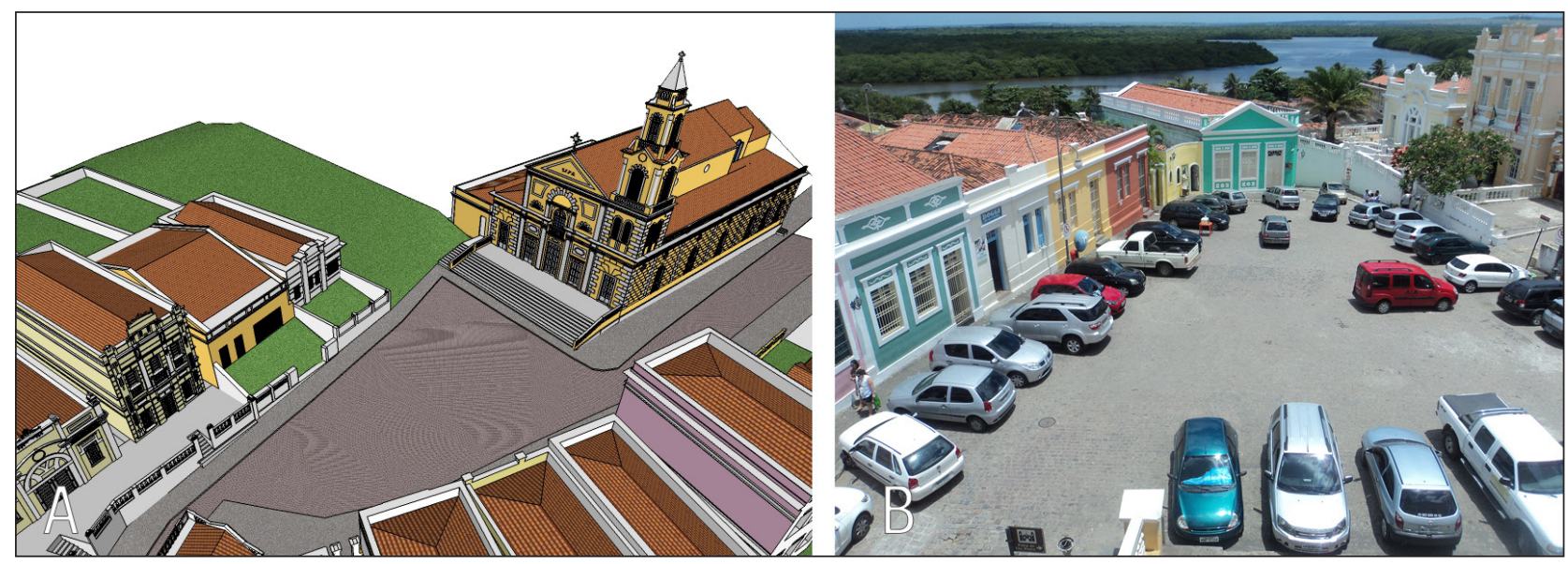

Figura 3 - Imagens do Largo de São Frei Pedro Gonçalves. (A) Vista aérea do modelo virtual tridimensional e (B) vista a partir da igreja Fonte: Elaboração própria. 
e o Cenário 2 como uma simulação de projeto de iluminação para o espaço estudado.

0 processo de criação dos cenários de realidade virtual foi desenvolvido com base na modelagem da geometria do espaço urbano e dos edifícios e no mapeamento básico de texturas e cores no programa Trimble SketchUp. Em seguida, o modelo foi exportado para o Autodesk 3DS Max, via extensão ".FBX”, para adequação da representação, tornando-a mais realista ao incluir iluminação dos cenários noturnos, sombras e mapeamento de texturas mais detalhados (Figura 4).

Cuidados foram tomados para uma melhor experiência no ambiente virtual, tendo em vista que procedimentos errados poderiam causar desconforto nos usuários. Os cenários foram renderizados pelo chamado "Render to Texture" no próprio programa, melhorando o desempenho de diversos processos em tempo real, considerando que tudo se transforma em um grande e único material. Os modelos renderizados foram então exportados para o Unity como extensão ".FBX", no qual foram criados os parâmetros para a visualização e interação dentro do ambiente virtual. Dispositivos auxiliares foram utilizados (Figura 5), como o controle bluetooth para navegação e o Google Cardboard ${ }^{\circledR}$ como head-mounted display (HMD) para permitir a imersão nos ambientes virtuais tridimensionais.

\section{Experimento}

Foram adotados três cenários: Cenário 0 , Cenário 1 e Cenário 2. 0 Cenário 0 foi admitido como referência para a configuração e ajuste das cores e propriedades das superfícies (transmissão e distribuição da luz) para os demais cenários. Uma vez que representa a situação diurna real com iluminação natural, o Cenário

\section{EketchUp}

Modelo tridimensional

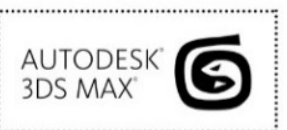

Mapeamento textura Iluminação Renderização

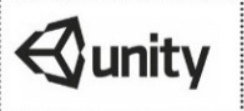

Interatividade

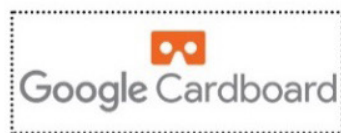

Head-mounted display

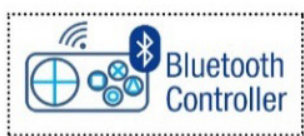

Navegação

Figura 4 - Workflow e recursos de imersão virtual do experimento

Fonte: Elaboração própria.

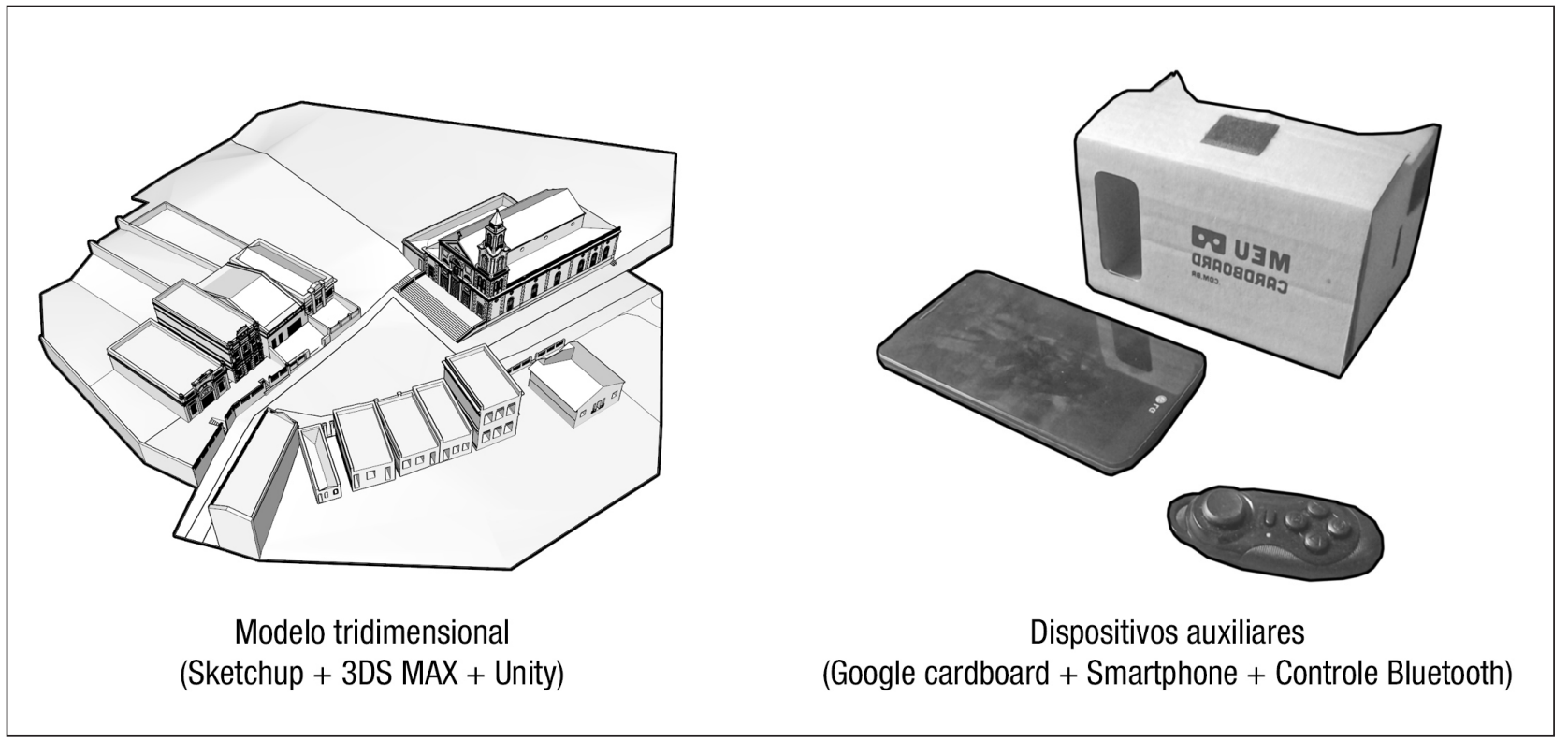

Figura 5 - Modelo tridimensional e dispositivos auxiliares utilizados no experimento Fonte: Elaboração própria. 
0 foi criado a partir de fotografias digitais do local, com objetivo principal de auxiliar na aproximação visual das cores e texturas das fachadas dos edifícios e pisos existentes que compõem o espaço urbano na área em estudo. Para a produção dos cenários com iluminação noturna (Cenário 1 e Cenário 2), utilizou-se o mesmo modelo produzido para o Cenário 0 , alterando-se apenas as condições de iluminação. A iluminação do Cenário 1 foi reproduzida a partir dos aspectos técnicos de temperatura de cor e intensidade das fontes luminosas existentes no local, de forma a torná-lo mais próximo possível da realidade. Quanto ao Cenário 2, partiu-se da proposta de um novo projeto de iluminação, utilizando-se do mesmo procedimento de modelagem do cenário anterior, tomando-se por base a temperatura de cor e a intensidade da proposta de intervenção no espaço (Figura 6).

Antes de iniciar o experimento, os participantes que foram convidados a utilizar o sistema receberam instruções do funcionamento, da navegação e da interação. Nenhum dos participantes relatou experiências anteriores no uso da tecnologia. Ao todo foram convidadas 12 pessoas, com idades variando de 15 a 44 anos, das quais seis pessoas eram do sexo feminino, e seis, do sexo masculino, além de a maioria (sete pessoas) declarar ser residente da cidade e conhecedor do local.

Do total de 12 participantes, duas pessoas participaram de testes preliminares a fim de verificar as orientações a serem dadas aos participantes, tempo por pessoa e questionário. Para tanto, foi realizado o seguinte procedimento: os participantes inseriam os óculos de realidade virtual para a apreciação do espaço, enquanto o pesquisador os guiava por meio da narração do que eles precisavam fazer. A informalidade da narração gerava trajetos diferentes nos três cenários, além de não existir um limite de tempo.

No momento de aplicação do questionário também não foi determinado um limite de termos a serem assinalados, o que causou contradições nos resultados preliminares. Essas ocorrências auxiliaram na otimização do processo de orientação ao usuário a partir da definição de um trajeto padrão, com ponto de partida ao lado da igreja (Figura 7). Dessa forma,

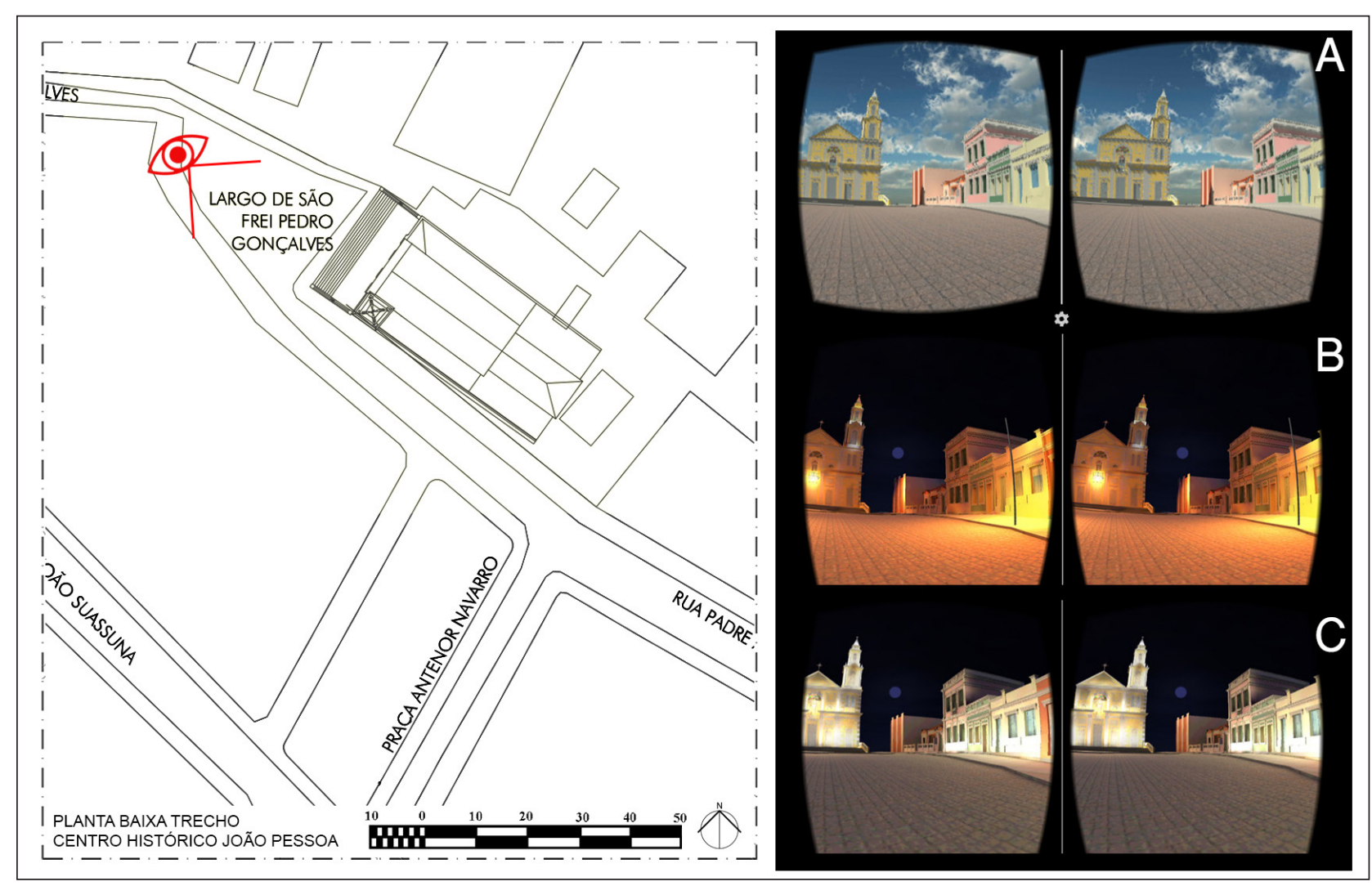

Figura 6 - Imagem do Largo de São Frei Pedro Gonçalves nos cenários descritos. Em planta baixa a indicação do ponto de vista. (A) Cenário 0, (B) Cenário 1 e (C) Cenário 2

Fonte: Elaboração própria. 
foi possível estipular o limite de tempo de 2 minutos na apreciação de cada cenário, para, em seguida, preencher o questionário, sendo definido o limite de até três termos entre seis alternativas. Esses testes preliminares foram descartados da análise final, restando dez avaliações a serem analisadas.

O período de realização do experimento durou três dias em novembro de 2015 na cidade de João Pessoa-PB. Foi escolhido um ambiente interno para a execução, no sentido de minimizar influências externas, como ruídos indesejáveis, que poderiam deturpar a avaliação do usuário. Levando isso em consideração, além da capacidade móvel do aparato tecnológico, o experimento foi realizado em dois locais: sala de aula no prédio de Arquitetura e Urbanismo da Universidade Federal da Paraíba (UFPB) e uma sala residencial no $12^{\circ}$ andar de edifício. Os ruídos urbanos eram minimizados ao fechar as esquadrias.

Ao longo do experimento, os usuários eram inseridos no ambiente virtual por intermédio do Cenário 0 como opção de treinamento, servindo de ajuda para se acostumarem com o aparato, com a navegação pelo ambiente e com a visualização dos espaços pelos óculos de realidade virtual, tendo como orientação as instruções dadas pelo pesquisador. Devido à possibilidade de visualização do cenário em $360^{\circ}$, os participantes preferiram ficar de pé.

Terminada a primeira fase de aprendizagem e do teste da tecnologia, foi solicitada aos usuários a remoção do HMD para descanso de 1 minuto.
Essa pausa está relacionada com a possibilidade de desconforto - tonturas e náuseas - causado pela longa exposição ao ambiente virtual por meio do HMD (Carvalho et al., 2011). Após essa fase, os participantes declararam não sentir incômodos provenientes da realidade virtual, e a navegação pelo ambiente foi considerada de fácil apreensão, semelhante a um videogame.

A próxima etapa foi a exploração do mesmo local, agora com cenários noturnos. Para isso, foi estabelecido o tempo limite de 2 minutos cronometrados, além do percurso básico para apreciação do espaço, no sentido de ter múltiplas perspectivas do local. Também foi comunicado aos usuários que os cenários seguintes seriam objeto de questionário a respeito da atmosfera percebida.

Assim, primeiramente foi inserido o Cenário 1, no qual os participantes deveriam caminhar até o final da ladeira - uma linha reta em direção à casa verde - e voltar para o mesmo local, ao lado da igreja (Figura 7). 0 mesmo procedimento foi então realizado no Cenário 2. Uma vez terminada a experiência nos três cenários, os participantes preenchiam o questionário.

A etapa do questionário envolveu questões relacionadas às impressões subjetivas do usuário no espaço em que ele foi inserido virtualmente. Portanto, a fim de compreender as impressões subjetivas dos ambientes estudados, foi desenvolvido o questionário a partir das dimensões de atmosfera percebida desenvolvidas por Vogels (2008). Levando em consideração o tempo para a exploração dos cenários e o preenchimento

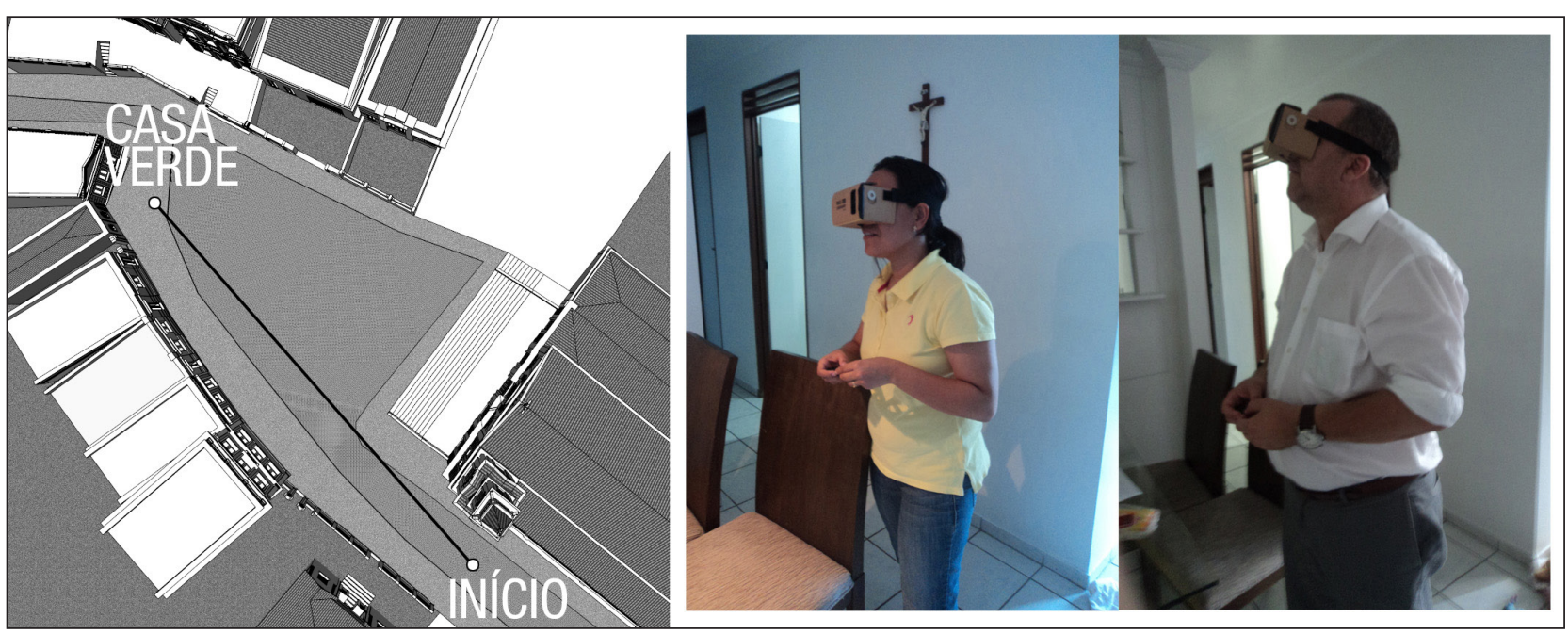

Figura 7 - Vista aérea do Largo de São Frei Pedro Gonçalves demonstrando o percurso básico de apreensão do espaço urbano. À direita, imagens dos participantes do experimento

Fonte: Elaboração própria. 
do questionário pelos participantes, foi necessária a redução da quantidade de termos, passando de 38 para 22, separados pelas quatro dimensões de atmosfera percebida, citadas anteriormente. Desses 22 termos, os participantes deveriam assinalar aqueles que melhor descrevessem suas sensações em cada um dos ambientes.

Para o questionário, foi previamente definido um limite de três termos, que poderiam ser marcados em cada dimensão, e, caso o participante não se sentisse confortável em responder ou não soubesse como descrever, ele poderia deixar em branco ou acrescentar alguma observação na pergunta ao final do questionário. Foram solicitadas também informações sobre os participantes, como a idade, o gênero, a familiaridade com o local e com a tecnologia utilizada. Vale a pena salientar que no experimento não foi dito que o foco do trabalho eram pesquisas qualitativas em iluminação, assim não foram induzidos a prestar atenção em aspectos particulares do ambiente.

\section{Análise e discussão dos resultados}

A partir da seleção dos termos que melhor descrevessem as dimensões de atmosfera percebida dos cenários estudados por parte dos participantes, foi possível visualizar tendências que, de acordo com as dimensões de Vogels, podem auxiliar no processo de design e decisões de projeto no espaço urbano.

Definidos em Cenário 1 e Cenário 2, respectivamente, ambiente com iluminação atual e ambiente com iluminação proposta, as dimensões de atmosfera percebida foram contabilizadas e comparadas entre os cenários por intermédio dos termos mais votados (Figura 8). Portanto, a primeira dimensão, aconchego (coziness), abrange termos que descrevem o ambiente como tranquilo, agradável, relaxante, romântico, seguro e acolhedor e demonstra alguns aspectos importantes a serem notados, por exemplo, a ausência da segurança como fator relevante no primeiro cenário, ao passo que se configura em um espaço tranquilo e romântico.

No caso dessa dimensão, o segundo cenário aparenta ser mais homogêneo e coerente, no qual o termo

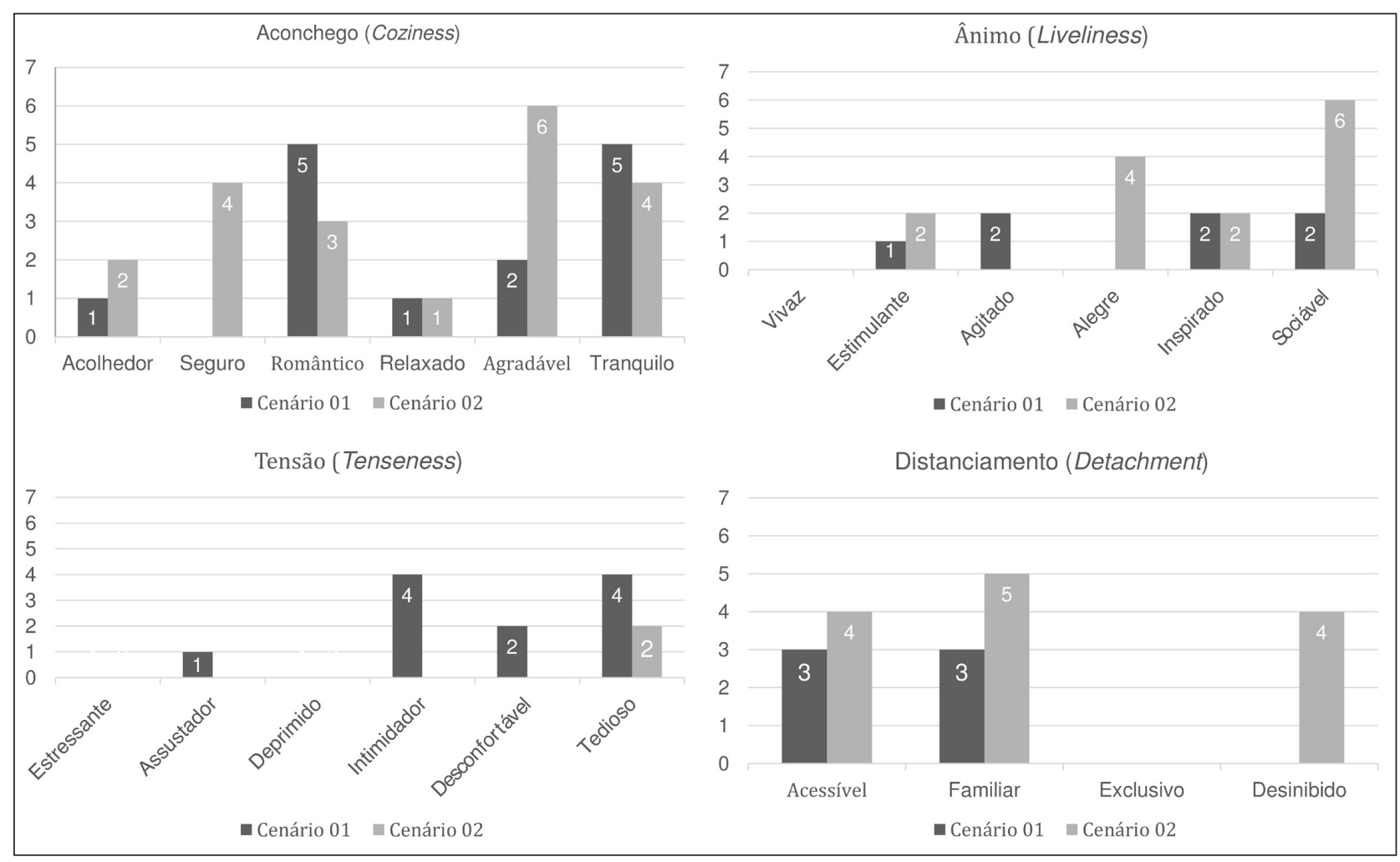

Figura 8 - Gráficos comparativos das dimensões aconchego, ânimo, tensão e distanciamento Fonte: Elaboraç̦ão própria. 
relacionado com a segurança aparece conjuntamente com a tranquilidade e, em menor grau, com o romantismo. Esse ambiente é considerado agradável do ponto de vista dos participantes devido à percepção visual de maior quantidade de luz existente entre esse cenário em comparação com o primeiro, o que justifica sua relação com segurança e confirma pesquisas a respeito da relação entre esses dois parâmetros (Fotios et al., 2005; Peña-García et al., 2015).

Segundo Casciani (2013), a iluminação urbana deverá mudar o aspecto da cidade contemporânea se estiver aberta à participação dos habitantes no processo de projeto, ao entender que as emoções e percepções dos usuários desempenham um papel importante na relação entre as pessoas e os espaços noturnos, devendo ser adequadamente estudadas. Quanto à dimensão ânimo (liveliness), o comparativo demonstra alguns aspectos relevantes relacionados com a capacidade do espaço em promover interações sociais e manifestações culturais.

0 termo "sociável" no Cenário 2 parece justificar essa teoria. 0 planejamento de iluminação formada pela composição de efeitos de luz coerente pode engajar os visitantes a permanecer no espaço, além de estimular outras funções, como o turismo e o comércio. Outro aspecto é a alegria no espaço. De acordo com o questionário, o Cenário 1 se apresenta como um espaço carente de condições que satisfaçam esse requisito, enquanto o Cenário 2 promove algo mais que transforma as qualidades percebidas do ambiente. Por vezes, os participantes descreviam verbalmente os ambientes por meio de questões estéticas, como bonito e feio, e comparativamente relacionavam aspectos, como socialização, segurança e romantismo.

As características da dimensão de tensão (tenseness) descrevem o Cenário 1 como um ambiente oposto ao Cenário 2, principalmente ao comparar com a dimensão de ânimo. Os termos "intimidador" e "desconfortável" provocam uma ligação indireta com a sensação de segurança, também relevante na primeira dimensão de análise, mas é contraditório ao entendê-lo como um espaço romântico, pois, se é intimidador e desconfortável, não deveria promover tal sensação.

O impacto do termo "tedioso" pode ser considerado como uma deficiência do ambiente virtual. Segundo os participantes, já que não foram inseridos elementos que favorecessem interações e movimentos, como pessoas e veículo, era declarado que no ambiente virtual faltava algo para fazer além de caminhar no espaço, o que pode ter influenciado a avaliação nos dois cenários estudados.

A última dimensão, distanciamento (detachment), tem aspectos relacionados ao comportamento e à impressão que o espaço subjuga o usuário. Os termos "acessível" e "familiar" são significativos em ambos os cenários, onde o primeiro seria característica inversa do termo "exclusivo", na medida em que é um espaço aberto a qualquer um, e no Cenário 2 , cuja iluminação foi tratada com cuidados de projeto, alcançou maior nível.

O segundo termo, familiar, no sentido de sensação de pertencimento, intimidade e respeito mútuo, foi considerado em maior grau no Cenário 2 em detrimento do Cenário 1. Aqui se conecta com alguns descritores da categoria aconchego, como romântico e agradável. Desinibição também tem um aspecto relevante no Cenário 2, pois gera uma relação direta com a capacidade sociável do espaço urbano, também marcante no mesmo ambiente, sugerido pela influência da iluminação na paisagem.

De maneira geral, o Cenário 2 demonstrou mais qualidades subjetivas positivas em relação ao Cenário 1, principalmente no tocante à segurança, e questões relacionadas à sociabilização e à tranquilidade do ambiente. As contradições identificadas - como a relevância de alguns termos díspares das dimensões aconchego e tensão, observadas no Cenário 1 refletem a complexidade e a importância de ajustar as avaliações para adequá-las à percepção do impacto da iluminação na paisagem urbana noturna.

\section{Limitações e contribuições}

A luz e suas ambiências estão intimamente ligadas à percepção que se tem dado ao espaço, seja ele externo, seja ele interno. 0 presente estudo procurou medir qualitativamente como a qualidade ambiental é percebida por meio de mecanismos, como a teoria da atmosfera percebida de Vogels, utilizando-se ambientes virtuais imersivos. Apesar de os participantes selecionarem termos que descreviam a avaliação afetiva esperada que os cenários propostos promoviam, alguns obstáculos e limitações foram encontrados.

A pesquisa procurou utilizar equipamentos com custos acessíveis e facilmente disponíveis, como smartphones e controles, cujo desempenho é notadamente menor 
se comparado com aparatos mais sofisticados e mais caros, como o Oculus Rift. É importante compreender as necessidades do experimento para a adequação da tecnologia. $\mathrm{O}$ objetivo de mapear qualidades ambientais tornou-se possível por intermédio da tecnologia, devido aos recursos de tempo e de custo disponíveis. Além da apreensão predominantemente visual e navegação exploratória, o aparato tecnológico possibilitava a execução do experimento em qualquer local, o que facilitou a participação de mais pessoas.

Entretanto, do ponto de vista técnico e operacional, alguns aspectos podem ser mais bem calibrados, principalmente quanto à interoperabilidade entre o software e o desempenho dos dispositivos de imersão e interação de realidade virtual. Exemplo dessas constatações é a navegação não otimizada via bluetooth. Uma sugestão seria utilizar pontos de vista predeterminados nos quais o usuário poderia ter uma visão 360 graus em vez da caminhada pelo ambiente. Outro fator foi o FPS (Frames per Second) baixo, causando movimentações de cabeça menos naturais, por causa da performance do dispositivo móvel utilizado (Google Cardboard). Durante o experimento, esse aspecto foi levado em consideração ao sugerir pausas entre cenários para descanso do participante. Procedimentos de melhorias e simplificações na geometria do espaço deverão favorecer uma melhor experiência.

0 presente artigo teve como contribuição um processo metodológico de mapeamento de impressões dos usuários utilizando ambientes virtuais tridimensionais. A interação dos participantes com os dispositivos utilizados e o desempenho de software e hardware foram essenciais para a condução do experimento e a validação da tecnologia e da metodologia. Também como contribuição está a avaliação de impressões por parte dos usuários ao utilizar três cenários distintos, tornando viável a utilização da ferramenta para esses fins, em prol de uma melhor paisagem noturna.

\section{Considerações finais}

A pesquisa apresentada no presente artigo versou sobre a importância da iluminação na qualidade percebida dos ambientes externos e também sobre conceitos relacionados à psicologia ambiental, com crescente interesse por parte dos pesquisadores no âmbito da iluminação, ajudando a compreender o papel que cada variável desempenha no espaço urbano, desde atributos luminotécnicos, como brilho, intensidade, distribuição e contraste, até características conceituais relacionadas com parâmetros ambientais, como mistério, coerência, ordem, clareza e complexidade.

A dificuldade de executar testes de luz em ambientes urbanos, devido, em parte, a entraves burocráticos, aliados ao custo elevado da produção de maquetes em escala real (mockups), justifica o uso de tecnologias computacionais no entendimento do espaço urbano iluminado.

Portanto, o desenvolvimento do experimento foi viabilizado ao utilizar-se de ambientes virtuais tridimensionais, cujo processo metodológico procurava criar cenários noturnos e compará-los por meio da atmosfera percebida, conceito desenvolvido por Vogels (2008). Assim, a ferramenta é relevante no sentido de superar obstáculos logísticos e financeiros em prol de uma solução alternativa para avaliação de projetos, levando-se em consideração as limitações decorrentes do uso da ferramenta e sua aceitação como opção de avaliação. Nesse aspecto, fatores citados anteriormente vão desde incômodos causados pela baixa qualidade da imagem, o próprio uso de equipamentos auxiliares, como o HMD no processo de imersão e de interação, até o grau de familiaridade em manusear esse aparato.

Os procedimentos adotados no experimento estão alinhados com a noção de planejamento participativo, extraindo contribuições qualitativas dos ambientes, cujo feedback deve ser compreendido como parte do processo de planejamento da iluminação pública, em uma abordagem centrada no usuário. Os resultados sugerem que ambientes iluminados de maneiras diferentes geram impressões qualitativas também diferentes, as quais podem impactar na dinâmica noturna de uma cidade e, portanto, precisam de melhor compreensão.

Neste trabalho, buscou-se mensurar apenas a percepção do usuário, e não sua participação ativa para intervir nos cenários apresentados, pois o enfoque da pesquisa foi obter um diagnóstico de cenários preconcebidos pelo olhar do usuário. Entretanto, considera-se como futuro desenvolvimento desta pesquisa a possibilidade de utilização das contribuições do usuário como auxiliadores do processo de projeto, introduzindo a capacidade de interação com opções que identifiquem os suportes de iluminação (luminárias de piso, aéreas ou de balizamento) e, assim, permitam a alteração do projeto original pelo usuário.

É importante salientar a aplicação da metodologia em outros contextos a fim de compreender o impacto 
do projeto na qualidade ambiental percebida, visando, consequentemente, à melhoria do produto final. No caso da habitação de interesse social, por exemplo, o baixo custo do aparato tecnológico possibilita que os espaços públicos sejam arranjados de diferentes maneiras e avaliados a partir da perspectiva do usuário. Particularmente importante, o envolvimento da comunidade no processo de projeto é uma das contribuições da metodologia, fornecendo os subsídios para que as intenções do projetista possam atender às expectativas dos usuários.

\section{Referências}

Angulo, A., \& Velasco, G. V. (2014). Immersive simulation of architectural spatial experiences. In Proceedings of the XVII Conference of the Iberoamerican Society of Digital Graphics (SIGraDi) (pp. 495-499). Valparaiso: Blucher.

Ball, J., Capanni, N., \& Watt, S. (2007). Virtual reality for mutual understanding in landscape planning. International Journal of Social, Behavioral, Educational, Economic, Business and Industrial Engineering, 1(11), 661-671.

Boyce, P., Eklund, N. H., Hamilton, B. J., \& Bruno, L. D. (2000). Perceptions of safety at night in different lighting conditions. Lighting Research \& Technology, 32(2), 79-91. http://dx.doi.org/10.1177/096032710003200205.

Bullinger, H., Bauer, W., Wenzel, G., \& Blach, R. (2010). Towards user centred design (UCD) in architecture based on immersive virtual environments. Computers in Industry, 61(4), 372-379. http://dx.doi.org/10.1016/j. compind.2009.12.003.

Carvalho, M. R., Costa, R. T., \& Nardi, A. E. (2011). Simulator Sickness Questionnaire: tradução e adaptação transcultural. Jornal Brasileiro de Psiquiatria, 60(4), 247-252. http:// dx.doi.org/10.1590/S0047-20852011000400003.

Casciani, D. (2013). Are citizens interested in their lit cities? A series of urban lighting impressions. In Proceedings of the 4th Professional Lighting Design Convention (pp. 5053). Copenhagen: VIA-Verlag.

Custers, P. J. M., Kort, Y. A. W., IJsselsteijn, W. A., \& Kruiff, M. E. (2010). Lighting in retail environments: atmosphere perception in the real world. Lighting Research \& Technology, 42(3), 331-343. http://dx.doi.org/10.1177/1477153510377836.

Cypriano, A. (2013). Iluminação artificial na percepção da arquitetura: considerações sobre aspectos quantitativos $e$ qualitativos no processo de projeto (Dissertação de mestrado). Faculdade de Arquitetura e Urbanismo, Universidade de São Paulo, São Paulo.

Drettakis, G., Roussou, M., Reche, A., \& Tsingos, N. (2007). Design and evaluation of a real-world virtual environment for architecture and urban planning. Presence, 16(3), 318332. http://dx.doi.org/10.1162/pres.16.3.318.

Dunston, P. S., Arns, L. L., Mcglothlin, J. D., Lasker, G. C., \& Kushner, A. G. (2011). An immersive virtual reality mock-up for design review of hospital patient rooms. In X. Wang \& J. J. Tsai (Eds.), Collaborative design in virtual environments (1. ed., pp. 167-176). Netherlands: Springer http://dx.doi. org/10.1007/978-94-007-0605-7_15.

Flynn, J., Hendrick, C., Spencer, T., \& Martyniuk, O. (1979). A guide to methodology procedures for measuring subjective impressions in lighting. Journal of the Illuminating Engineering Society, 8(2), 95-110. http://dx.doi.org/10.1080/009944 80.1979.10748577.

Flynn, J., Spencer, T., Martyniuk, O., \& Hendrick, C. (1973). Interim study of procedures for investigating the effect of light on impression and behavior. Journal of the Illuminating Engineering Society, 3(1), 87-94. http://dx.doi.org/10.10 80/00994480.1973.10732231.

Fotios, S., Cheal, C., \& Boyce, P. (2005). Light source spectrum, brightness perception and visual performance in pedestrian environments: a review. Lighting Research \& Technology, 37(4), 271-291. http://dx.doi.org/10.1191/1365782805li139oa.

Gonçalves, A. L. A. (2005). Iluminação urbana de conjuntos históricos e tradicionais: adequação do projeto à ambiência: Uma metodologia para planos diretores de iluminação: o caso do bairro histórico de Paraty (Tese de doutorado). Faculdade de Arquitetura e Urbanismo, Universidade de São Paulo, São Paulo.

Heydarian, A., Carneiro, J. P., Gerber, D., \& Becerik-Gerber, B. (2015a). Immersive virtual environments, understanding the impact of design features and occupant choice upon lighting for building performance. Building and Environment, 89, 217-228. http://dx.doi.org/10.1016/j.buildenv.2015.02.038.

Heydarian, A., Carneiro, J. P., Gerber, D., Becerik-Gerber, B., Hayes, T., \& Wood, W. (2015b). Immersive virtual environments versus physical built environments: a benchmarking study for building design and user-built environment explorations. Automation in Construction, 54(7), 116-126. http://dx.doi.org/10.1016/j.autcon.2015.03.020. 
Hong, O. S. (2007). Design basis to quality urban lighting masterplan (Dissertação de mestrado). School of Design \& Environment, National University of Singapore, Singapore.

Kaplan, S. (1992). Perception and landscape: conceptions and misconceptions. In J. L. Nasar (Ed.), Environmental aesthetics: theory, research and applications (1. ed., pp. 45-55). New York: Cambridge University Press.

Knight, C. (2010). Field surveys of the effect of lamp spectrum on the perception of safety and comfort at night. Lighting Research \& Technology, 42(3), 313-329. http:// dx.doi.org/10.1177/1477153510376794.

Lindh, U. W. (2012). Light shapes spaces: experience of distribution of light and visual spatial boundaries (Tese de doutorado). Faculty of Fine, Applied and Performing Arts, University of Gothenburg, Gothenburg.

Llorach, G., Evans, A., \& Blat, J. (2014). Simulator sickness and presence using HMDs: comparing use of a game controller and a position estimation system. In Proceedings of the 20th ACM Symposium on Virtual Reality Software and Technology (pp. 137-140). Edinburgh: ACM.

Maing, M. (2012). Virtual mock-up modeling as study model of building envelope, performance and design. In Proceedings of the Simbuild 2012 (pp. 75-82). Wisconsin: IBPSA.

Mascaró, L. (2006). A iluminação do espaço urbano (1. ed.). Porto Alegre: Masquatro.

Peña-García, A., Hurtado, A., \& Aguilar-Luzón, M. C. (2015). Impact of public lighting on pedestrians' perception of safety and well-being. Safety Science, 78(8), 142-148. http://dx.doi.org/10.1016/j.ssci.2015.04.009.

Portman, M. E., Natapov, A., \& Fisher-Gewirtzman, D. (2015). To go where no man has gone before: virtual reality in architecture, landscape architecture and environmental planning. Computers, Environment and Urban Systems, 54, 376-384. http://dx.doi.org/10.1016/j. compenvurbsys.2015.05.001.

Sá, A. G., \& Zachmann, G. (1999). Virtual reality as a tool for verification of assembly and maintenance processes.
Computers \& Graphics, 23(3), 389-403. http://dx.doi. org/10.1016/S0097-8493(99)00047-3.

Skarlatou, A. (2010). Light effects in the design process: a theoretical investigation of designers' perceptions of light effects and an empirical study of how they use them in architectural lighting design (Tese de doutorado). Faculty of the Built Environment, University College London, London.

Tori, R., \& Kirner, C. (2006). Fundamentos de realidade virtual. In R. Tori, C. Kirner, \& R. Siscouto (Eds.), Fundamentos e tecnologia de realidade virtual e aumentada: livro do pré-simpósio VIII Symposium on Virtual Reality (1. ed., pp. 2-21). Belém: SBC.

Veitch, J. A. (2001). Psychological processes influencing lighting quality. Journal of the Illuminating Engineering Society, 30(1), 124-140. http://dx.doi.org/10.1080/009 94480.2001 .10748341$.

Vogels, I. (2008). Atmosphere metrics: development of a tool to quantify experienced atmosphere. In J. H. D. M. Westerink, M. Ouwerkerk, T. J. M. Overbeek, W. F. Pasveer, \& B. Ruyter (Eds.), Probing experience: from assessment of user emotions and behaviour to development of products (Vol. 8, pp. 25-41). Netherlands: Springer.

Whisker, V. E., Baratta, A. J., Yerrapathruni, S., Messner, J. I., Shaw, T. S., Warren, M. E., \& Rotthoff, E. S. (2003). Using immersive virtual environments to develop and visualize construction schedules for advanced nuclear power plants. In In Proceedings of the 2003 International Congress on Advances in Nuclear Power Plants. Córdoba: ICAPP.

Yan, W., Culp, C., \& Graf, R. (2011). Integrating BIM and gaming for real-time interactive architectural visualization. Automation in Construction, 20(4), 446-458. http://dx.doi. org/10.1016/j.autcon.2010.11.013.

Recebido: Ago. 23, 2016

Aprovado: Fev. 23, 2017 